\title{
In-Patient Psychotherapy at the Cassel Hospital
}

\author{
J. D. Denford, Consultant Psychotherapist, The Cassel Hospital, Richmond, Surrey
}

\begin{abstract}
Admission to hospital for psychotherapy facilitates communication with patients and allows more ways of influencing them than do conventional out-patient situations. Small and large groups can be added to individual interviews, and living together allows the development of many potentially therapeutic relationships with other patients and staff. This additional influence can be ignored. If it is assumed to be an integral part of treatment and organised rationally, the whole hospital becomes its instrument; psychotherapists, nurses, patients, domestic staff and administrators can be seen to be subordinate to that whole, and their traditional activities, attitudes to each other, and theories, are inevitably modified. Traditional boundaries between the roles of different workers become blurred, while how they get on with each other has important consequences for patients, so that their separate roles and functions must be clearly defined. ${ }^{1.2}$
\end{abstract}

\section{Treatment structures}

At the Cassel Hospital, which was founded in 1919 as a private institution by Sir Ernest Cassel and entered the NHS in 1948, understanding of these matters and organisation to accommodate them were developed by T. F. Main, D. Weddell and others between 1945 and $1976,{ }^{3}$ and are still changing. The hospital organisation provides areas for discussion and other action where observations can be made; situations where formulation of those observations in useable forms can be developed; opportunities for integration of the two main classes of formulation - the ideational and practical-into meaningful wholes; their dissemination to all groups of staff and patients; and the transmission in a reverse direction, back to patients, of formulations that reflect the various degrees of reality-testing available in the hospital, and in the outside world. Administration and research are linked with this organisation and make contributions to treatment from their different positions.

\section{An implied contract}

Reasonable control of living conditions is essential if psychotherapy is to be carried on. Limits within which patients are expected to confine their activities are clearly defined, and amount to unwritten contracts between staff and patient which allow ready recognition of acts which exceed them. They are determined by the principles of consideration for others in a community balanced against reasonable needs for personal freedom, acceptance of the responsibilities for oneself that being a voluntary patient undertaking psychotherapy implies, and for the treatment of others that having psychotherapy in a group implies.
Patients are expected to participate in therapeutic activities of all kinds: to stick to the timetable, especially getting up from and going to bed; to come to meals; to work at expected tasks, e.g. cleaning and caring for their parts of the hospital, and helping in the planning, ordering and cooking of certain meals (breakfasts, and evening and weekend meals). Under supervision, on certain special occasions, they sign the leave book when they go out of the hospital. They are required to hand over all drugs to the staff, pay for breakages where reasonable; undertake to spend weekends at home after the first one after admission where this is possible, and so on. They are expected not to smoke in non-smoking areas, especially bedrooms, and not to drink alcohol in the hospital except with nurses' agreement.

Nurses have the chief responsibility for practical matters. They develop, sustain and share work and social activities of all kinds. They use encouragement, understanding of difficulties in work and social terms, and honesty about both their own feelings and those of patients to facilitate functioning. Inevitably they develop relationships with patients and these are assumed to constitute important elements of what patients internalise in the course of their stay.

\section{Unit arrangements}

The hospital has three in-patient units (as well as a small school, a day unit for children, and out-patients), two for single adults, and one for families and adolescents. Unit staff are nurses and nursing officer, therapists and consultant, administrator and secretary, who meet daily both with patients and among themselves. There is also a community team of doctors and nurses whose responsibilities are whole hospital matters, and who meet all patients twice weekly and monitor unit activities. Once a week all professional staff meet to discuss difficulties arising from the work.

Patients also have their officers, both for units and for the whole community, elected by themselves (with nurse advice) for limited terms, who chair meetings and represent patients to staff.

Management of practical matters takes place formally at regular unit and community meetings in which all patients are encouraged to join. Much real work and responsibility for maintenance of the hospital fabric and furniture can be given to patients because it has never been policy to employ more than very small numbers of maintenance and cleaning staff. Instead a sum of money (the therapeutic budget) is allocated by the District Health Authority each year to service such work carried out by patients. Each unit is given a 
sum to support their cleaning, refurnishing and maintenance of fixtures, etc. with the expectation that limits will be kept and money accounted for. Purchases are made on joint nurse/patient expeditions. There is the ever-present possibility of failure-with discomfort, and opprobium from fellow-patients as consequences. Many patients have had no previous experience of managing money or related practical matters. Similar therapeutic opportunities regularly recur in the planning, ordering and preparation of food. Significant economies result from these practices.

The formal psychotherapy patients receive varies between different units. Some have twice or even thrice weekly individual interviews with their therapist, others meet him once alone and twice in a small group with his other patients each week. Patients in each unit meet their nurses and consultant as a group each day for discussion of unit matters, and the whole hospital's patients meet the community team twice a week to attend to matters affecting them all. Patients also attend other regular meetings concerned with particular matters such as catering, the nights, and weekends. Impromptu meetings may be called to deal with crises.

\section{Discussion}

Three agents of therapeutic effect may be identified: the living together, the nursing, and formal psychotherapy. This may also be the order of their importance.

\section{Living together}

The patient culture may be the primary and ultimate therapeutic instrument, and the staffs tasks to prescribe, construct and maintain it, making practical or ideational contributions to it through the various channels of communication available. Patients spend much longer with each other than with any staff and are in a position to make significant contributions to each other's treatment. Most have conflicts about parents and authority and often accept influence more easily from sibling-like relationships, especially as their resistances are mobilised by the parent-like staff. ${ }^{4}$

The hospital occupies an old building that has been a private home, a school, and an hotel. It required constant work to keep it going. The absence of single bedrooms makes rooming issues a repeated challenge to adaptation. The same can be said about the number and nature of patients in the hospital; when the hospital is full work goes better though patients feel crowded.

Patients who present challenges that are not beyond the available resources stimulate progress in others. Neither for patients nor staff is it a comfortable or easy situation, but it is clearly not desirable that it should be for those who have the capacity to respond. For patients who do not have such capacities, however, the difficulties are too great. Those who tend to break down into psychotic states generally get worse and are not suitable. A relatively drug-free culture has many advantages in helping those who have become dependent, but is another reason for excluding patients whose conditions require drug treatment. Attitudes to smoking - people do not smoke in most groups, or in the servery, dining room or bedrooms-influence individuals through the culture. The value of physical exercise has also become established through the introduction to the programme of regular twice-weekly runs or walks for both staff and patients and can be seen to influence a proportion of patients (and staff). Because the intention has been to establish in hospital some home characteristics it is inevitable and desirable that people should have and preserve some degree of privacy. Psychotherapy involves the penetration of that privacy and much of the machinery of the hospital has been developed to protect individuals from too disturbing or hurtful consequences. The main cultural element that seeks to achieve this is the attitude of serious respect and attention given to all private material. It is assumed that all who should know particular things for their own treatment or safety should learn of them, but there is also an expectation that such private knowledge should be accorded the same care as if it were one's own.

There is not a hospital structure or circumstance that could be described or developed that may not be perverted by patients at some time or another for the purpose of maintaining their habitual avoidances of anxiety. Patients bring their habitual behaviours in with them, and much of the treatment work consists of identifying and resisting the perversions of structures which are their consequence. Freedom to choose room-mates may be exploited to sustain social isolation, privacy may be exploited to sustain secret drinking or drug-taking, becoming patient chairman may be used to avoid facing emotional issues essential if treatment is to proceed. Such distortions are important elements in the private life that patients sustain both individually and in common. Treatment is designed to reach into this private life to gather understandings of what is there, and to bring them back into the sphere of social reality to attempt some joining between the fantastic and private and the real and social. Private fantasies and their accompanying behaviour have developed in isolation. Neurotic anxieties reflect a child's view of the world rather than that of a matter-of-fact adult. Therapeutic effects should result from the drawing of such private elements into touch with the reality of others' thoughts and feelings.

\section{Nursing, and formal psychotherapy}

Patients are admitted for an initial trial period of one month following selection on the basis of out-patient consultation and home visits by a nurse and patient. The nurse's visit is as significant in assessment as the consultation with the doctor.

Patients who show indications, however slight, of being able to use the living situation and formal psychotherapy to some benefit, are accepted. Unsuitable patients are the psychotic or those liable to break down into such states under pressure, those addicted to alcohol or drugs (whose addiction we cannot control) and those whose disturbance leads to behaviour, whether violent or distorted, which is beyond the resources of our small staff to contain. (Some patients 
may benefit from psychotherapy only in an environment which can control their actions.) Attempts are made to exclude clearly unsuitable referrals before interviews, because attachment to the hospital and involvement in relationships begins with these first personal contacts and can make refusal disturbing or destructive.

If a decision is made to treat and the offer accepted there follows a period of roughly 9-15 months within which therapeutic processes can take place. In regular and individual and group interviews, in the day-to-day work and recreation periods, and in all the negotiations arising from evenings and nights, weekends, holidays, practical and financial arrangements in their lives, relations with families and friends outside the hospital and with all manner of official agencies, therapists and nurses working from their respective positions build up complementary pictures of the patient's personality and its functioning, his inhibitions, strengths and weaknesses, and the ways in which deficiencies and distortions in his earlier experiences determine aberrations in his present perceptions and behaviour. A steady effort is made to relate therapist and nurse formulations with each other so as to avoid the development of staff splitting which can be disturbing to patients, and to allow each view to inform the therapeutic effort of the other worker. Therapists are able to increase the immediacy and accuracy of their interpretations and discussions of mental content because they know practical things, and nurses to modify their practical interventions and ways of accompanying patients because of their understanding of what the therapist has gleaned. They can resist dependency and other manipulations that might allow patients to continue avoidances of anxiety-provoking situations, which it is the ultimate aim of treatment to reduce or eliminate. Patients are presented with not just individual relationships with nurse and therapist to internalise, but also a sense of their coherent relationship with each other and of their being part of a larger hospital structure, all of which have significant and powerful meanings of reassurance and reliability. Long-term follow-up shows that many patients retain a sense of continuing relationship with a complex hospital entity as a useful source of confidence and conviction about themselves.

Issues of eventual termination and leaving are focussed on from the beginning, as ways of countering dependency and facilitating the grieving that is assumed necessary if this is to be accomplished without nullifying the positive effects of the treatment experience. An analysis of the processes of treatment is contained in Denford et al. ${ }^{5}$

Giving as much responsibility as possible to patients, or having as much as possible at hospital rather than district level, has inevitably conflicted with the centralisation of management brought about by the 1974 reorganisation of the NHS. The treatment arrangements described attempt to maintain in a hospital certain significant elements that are naturally present in a family home. The therapeutic budget, money we can call our own and for which we can be responsible, protects our treatment structures in the same way as a salary allows a family's autonomy.

\section{The nature of treatment}

The hospital's work is understood as observing patients' behaviour, making formulations about it relative to all the factors understood determining its nature, and conveying these more coherent ideas back to patients, both in words and actions, with the hope of modifying that behaviour in more rational and appropriate directions. Therapeutic effects are assumed to result from learning processes whereby such ideas are absorbed both as themselves and as elements in relationships with individuals-patients and staff-groups of various sizes, and the complex object of the whole hospital. Such learning is facilitated by the organisation's being supportive, firm but flexible, coherent and generally rational. Kernberg ${ }^{6}$ has discussed the application of Rice's systems theory to the therapeutic structure of hospitals like the Cassel, and how treatment can be conceived of as a 'form of (conscious or unconscious) learning of leadership of one's own self. Parallels with the structure of well-functioning families are obvious.

There is an assumption of a conflict existing between the perceptions, formulations and responses anyone can have regarding a patient with whom he has a personal connection, and the equivalent impressions of an independent observer. Hospital structures assume that there are unique but complementary values in both views, each essential to the practice of psychotherapy. The organisation is a mechanism to achieve a realistic and tolerable (to the patient, staff and the world outside hospital) balance between these two, not only to create and maintain suitable circumstances for psychotherapy, but also as a target of treatment.

\section{The patients}

Our methods have been developed to treat more severely neurotic patients, who do not need the complete assumption of responsibility by the professional a psychotic patient requires, but do need more than is possible with outpatients. People whose treatment is possible as out-patients may be sufficiently integrated to do enough reality testing work or 'Control' function ${ }^{6}$ themselves, to make progress without the therapist needing to extend his areas of observation. If more disturbed neurotic patients are to be treated at all they appear to need some such team organisation as this. Most of them have presented treatment problems beyond the resources of individual therapists, or of hospitals without a coherent organisation, or, if they can be treated by the usual means, it takes too long. Because they are so difficult to treat successfully and because they so often come from poor family backgrounds, private treatment is beyond their means so the NHS has them (usually making severe demands on scarce resources). People must be trained to do such work.

\section{The staff}

Intense and difficult therapeutic relationships develop with neurotic and borderline individuals, particularly when treatment avoids the use of tranquillising emotionally 
suppressive drugs. Such people use projective mechanisms often of powerful aggressive elements in their thoughts, to such an extent that therapists and others in contact with them become confused and disturbed themselves and need both outside help with their own testing of reality regarding the patient, and help themselves, in their own right, with the feelings that arise because of the aggression they are shown. A clearly structured and cohesive staff structure provides most effective support. Staff must talk regularly and honestly with one another about the feelings and conflicts that inevitably arise both to be an efficient team, but also to provide effective mutual support. Professional behaviour becomes less distorted by patient psychopathology and so more reasonable.

Nurses are most directly exposed to patients, for the longest periods and under the least controllable conditions; the same is true for some domestic staff. Therapists meet different pressures but their circumstances are controllable and periods of exposure shorter. They are usually in psychoanalytic or some parallel training and their own therapies are potent sources of help, but adequate and regular supervision is essential. Again, nurses are much less often in psychotherapy of their own and so must be helped by the hospital. Whatever its source, support in the work is assumed to come from adequate understanding of the psychological processes of all sorts that are occurring in both patients and workers.

\section{After-care and follow-up}

Patients are passed back to referrers for any necessary further supervision or treatment. The hospital has not had the resources to provide continuing supervision. It will however offer advice about further psychotherapy where this is wanted and seems justified. Follow-up was regularly done between 1921 and 1948 but became only occasional thereafter. Rayner \& $\mathrm{Hahn}^{7}$ did a postal follow-up, and a group of families was studied by Biggs. ${ }^{4}$ Changes in drug use after treatment were followed up by Lanham. ${ }^{8}$ Recently the group of patients whose treatment was reported in Denford $e t a l^{5}$ have been followed up five years after discharge (to be published) showing considerable gains apparently as a result of treatment. For such severely disabled people the period of treatment practically available is usually and not surprisingly insufficient. Most need further help. Many will need some sort of psychiatric supervision all their lives, but successful treatment has improved the quality of their lives. Many seem to become more able to use out-patient treatment, including psychotherapy, and need fewer admissions. Patients often revert to their pretreatment states of demoralisation around discharge, but re-achieve gains some time after. It may be that the most general and consistent effect of treatment is the substitution of hopeful and optimistic attitudes, and increased readiness to try to achieve things in life, for the generally hopeless and pessimistic attitudes and the apathy that are characteristic before admission.

\section{REFERENCES}

${ }^{1}$ JAMES, O. \& WILSON, A. (1981) A theoretical basis for therapeutic community work. Unpublished presentation to Cassel Hospital staff.

${ }^{2}$ JAMES, 0 . (1984) The role of the nurse-therapist relationship in the therapeutic community. International Review of Psychoanalysis, 11, 151-160.

${ }^{3}$ BARNES, E. (ed.) (1968) Psychosocial Nursing. London: Tavistock.

'BIGGs, V. (1980) Inpatients' view of the Cassel experience. Paper read at the Cassel Hospital Jubilee Conference.

${ }^{5}$ Denford, J., SCHAChTER, J., TeMple, N., Kind, P. \& Rosser, R. (1983) Selection and outcome in inpatient psychotherapy. British Journal of Medical Psychology, 56, 225-243.

${ }^{6} \mathrm{KeRNBERG}$, O. (1976) Towards an integrative theory of hospital treatment. In Object-Relations Theory and Clinical Psychoanalysis, New York: Aronson.

'RAYNER, E. \& HahN, H. (1964) A follow-up of Cassel patients. Unpublished presentation to Hospital staff.

${ }^{8}$ LANHAM, W. R. (1979) Psychotropic drug prescription to neurotic patients after discharge from a drug-free hospital. M.Sc. dissertation, University of Surrey.

\section{The Merck, Sharp and Dohme Prize in Psychiatry}

The winner of the 1986 Merck, Sharp and Dohme Prize in Psychiatry (East Anglia) was Dr Susan Thomas, Registrar, Fulbourn Hospital, Cambridge with an entry on 'The Contribution of Computed Axial Tomography to Child Psychiatry: A Critical Review'.

The Academic Department of Psychiatry announces that the competition has now been opened for the 1987 MSD Prize: First Prize £150, and Second Prize $\mathbf{1 4 0}$. Psychiatric Trainees working in the East Anglian Region are eligible. For details, please contact: Mrs M. A. Coburn, PGME Senior Secretary, Fulbourn Hospital, Cambridge CBI 5EF (telephone: Cambridge 248074, extension 261).

\section{New Computer System}

A computerised Patient Administration System (PAS) has recently been installed at Napsbury Psychiatric Hospital, near St Albans. It was designed by Protechnic Computers Ltd of Cambridge and is capable of providing the doctor, community nurse and social worker with clinically essential knowledge of the patient's medical history which may be needed quickly in a crisis. The PAS will hold information on drugs prescribed and other developments include patients' banking, which is important in establishing the independence of long-stay patients who may need to re-learn how to use money as preparation for leaving hospital. 